\title{
Burkholderia cenocepacia utilizes ferritin as an iron source
}

Correspondence

Terrence L. Stull

tstull@ouhsc.edu

Received 10 June 2005

Accepted 16 February 2006

\author{
Paul W. Whitby, ${ }^{1}$ Timothy M. VanWagoner, ${ }^{1}$ Jennifer M. Springer, ${ }^{1}$ \\ Daniel J. Morton, ${ }^{1}$ Thomas W. Seale ${ }^{1}$ and Terrence L. Stull ${ }^{1,2}$ \\ Departments of Paediatrics ${ }^{1}$ and Microbiology/Immunology ${ }^{2}$, University of Oklahoma Health \\ Sciences Center, Oklahoma City, OK 73104, USA
}

\begin{abstract}
Burkholderia cenocepacia is a member of the Burkholderia cepacia complex, a group of genetically similar species that inhabit a number of environmental niches, including the lungs of patients with cystic fibrosis (CF). To colonize the lung, this bacterium requires a source of iron to satisfy its nutritional requirements for this important metal. Because of the high potential for damage in lung tissue resulting from oxygen-iron interactions, this metal is sequestered by a number of mechanisms that render it potentially unavailable to invading micro-organisms. Such mechanisms include the intracellular and extracellular presence of the iron-binding protein ferritin. Ferritin has a highly stable macromolecular structure and may contain up to 4500 iron atoms per molecule. To date, there has been no known report of a pathogenic bacterial species that directly utilizes iron sequestered by this macromolecule. To examine the ability of ferritin to support growth of $B$. cenocepacia J2315, iron-deficient media were supplemented with different concentrations of ferritin and the growth kinetics characterized over a $40 \mathrm{~h}$ period. The results indicated that $B$. cenocepacia J2315 utilizes iron bound by ferritin. Further studies examining the mechanisms of iron uptake from ferritin indicated that iron utilization results from a proteolytic degradation of this otherwise stable macromolecular structure. Since it is known that the ferritin concentration is significantly higher in the CF lung than in healthy lungs, this novel iron-acquisition mechanism may contribute to infection by $B$. cenocepacia in people with CF.
\end{abstract}

\section{INTRODUCTION}

The Burkholderia cepacia complex (Bcc) contains nine genetically distinct but phenotypically similar species (Mahenthiralingam et al., 2005). In addition, the Bcc also contains a number of genomovars for which a designated species name has yet to be assigned, pending definitive biochemical tests (Mahenthiralingam et al., 2005). Originally identified as phytopathogens (Burkholder, 1950), this group of organisms has become a focus of attention as human pathogens following isolation from the respiratory tracts of patients that are immunocompromised or have cystic fibrosis (CF) (Rosenstein \& Hall, 1980; Isles et al., 1984; Thomassen et al., 1985). In the early 1980s, the incidence of B. cepacia infection in CF clinics increased dramatically. This, coupled with factors such as the innate resistance of the organism to multiple antibiotics, poor clinical prognosis, and high degree of person-to person transmission, led to fundamental changes in both hospital infection control policies and the social behaviour of CF patients (Govan et al., 1993; Walters \& Smith, 1993; Lewin et al., 1993; Prince, 1986;

Abbreviations: AEBSF, 4-(2-aminoethyl)benzenesulfonyl fluoride; Bcc, Burkholderia cepacia complex; CF, cystic fibrosis; EDDHA, ethylenediamine- $N, N^{\prime}$ bis(o-hydroxyphenyl)acetic acid.
Simpson et al., 1994). While initial attempts to stem the increasing prevalence of Bcc infections involved cohorting infected patients, complete segregation of all patients is becoming the norm (Vonberg \& Gastmeier, 2005). This has resulted in a significant decrease in the acquisition of these pathogens. However, new infections still occur and suggest an environmental reservoir of the Bcc.

While representatives of all nine Bcc species have been isolated from CF patients, B. cenocepacia is the major CF pathogen (Mahenthiralingam et al., 2005). Within this species there are several highly clonal lineages, of which one, ET12, was responsible for epidemics across the northern USA and Europe in the 1990s (Govan et al., 1993; Johnson et al., 1994; Pitt et al., 1996). Infection with an isolate from the ET12 lineage is associated with poor prognosis consistent with 'cepacia syndrome', a rapid and fatal decline in lung function characterized by a necrotizing pneumonia with fever, bacteraemia, elevation of erythrocyte sedimentation rate and leukocytosis (Isles et al., 1984). While there have been numerous reports detailing various potential virulence factors, such as proteases, haemolysins, pili and siderophores (Kooi et al., 1994; Nakazawa et al., 1987; Hutchison et al., 1998; Sokol, 1986; Darling et al., 1998), the specific molecular mechanisms that allow this pathogen to 
proliferate in the CF lung, but not healthy lungs, have yet to be elucidated. Presumably, the ability to colonize the $\mathrm{CF}$ lung relates to a physiological aspect of the environment favouring growth, such as the nutritional availability of an essential moiety. Our previous experience studying the haem and iron acquisition of Haemophilus influenzae, also a CF lung pathogen, suggests that iron availability may play a role in the colonization of the CF lung by $B$. cenocepacia. While it is known that B. cenocepacia produces siderophores, the role of the siderophores in obtaining iron from physiological iron sources to support growth in humans has not been examined. Our laboratory has identified and characterized a potentially secreted RTX toxin that may play a role in cytolysis (Whitby et al., 2006), releasing intracellular iron sources that may satisfy the demand for iron.

Iron metabolism of the lung differs markedly from that of the rest of the body (Gutteridge et al., 2001). Due to the increased possibility of iron-mediated oxidative damage to tissues at this site, free iron is rapidly sequestered and removed. Each day, the respiratory tract of humans is exposed to approximately $10 \mu \mathrm{g}$ of soluble, airborne iron (Turi et al., 2004). The risk of damage to the lung is further enhanced by the high partial pressure of oxygen and presence of inhaled micro-organisms. The main proteins involved in iron sequestration include transferrin, lactoferrin and ferritin. In the CF lung, ferritin is significantly upregulated in comparison with normal healthy lungs (Stites et al., 1998, 1999; Turi et al., 2004). Ferritins are a superfamily of roughly spherical, shell-like iron-storage proteins that are ubiquitous in both prokaryotes and eukaryotes. In higher organisms, each molecule consists of 24 subunits assembled into a hollow sphere with numerous pores allowing iron ingress and egress. The subunits can be either heavy or light ( $\mathrm{H}$ or $\mathrm{L}$ type), having molecular masses of $\sim 21$ or $\sim 19 \mathrm{kDa}$, respectively (Liu \& Theil, 2005). The ferritins from individual tissues vary with respect to the individual number of H- or L-type subunits. Each subunit joins to its neighbouring subunits through non-covalent interactions and packs to form a hollow sphere $\sim 8 \mathrm{~nm}$ in diameter, with walls that are $\sim 1 \mathrm{~nm}$ thick and a combined molecular mass of approximately $474000 \mathrm{kDa}$. Each ferritin molecule can hold approximately 4500 iron atoms, either as a sequestered form of iron or as a bioavailable store for later utilization. As such, ferritin represents a concentrated source of iron for the growth of micro-organisms. However, the protein is extremely stable, being resistant to $\mathrm{pH}$ levels as low as $2 \cdot 0$, as well as being highly thermostable and resistant to most proteases (Crichton, 1969).

Previous investigations of lung iron metabolism have shown that the CF lung has a very high ferritin level. In the study of Stites et al. (1999), ferritin levels in bronchial alveolar lavage (BAL) specimens of healthy humans were $\sim 9 \mathrm{ng} \mathrm{ml}^{-1}$, while in CF patients, the mean BAL ferritin level was 70-fold higher (mean $647 \mathrm{ng} \mathrm{ml}^{-1}$, range $200-1200 \mathrm{ng} \mathrm{ml}^{-1}$ ). In contrast, there was no apparent increase in transferrin levels between the two groups in the study. In a separate study by
Reid et al. (2004), evaluating the complex role of ironregulatory cytokines and iron metabolism in the CF lung, CF sputum ferritin was recorded as ranging between 890 and $6980 \mathrm{ng} \mathrm{ml}^{-1}$. A significant increase in ferritin levels was associated with infection of the CF lung by Pseudomonas aeruginosa. This study indicated that both the proinflammatory cytokine IL- $1 \beta$ and TNF- $\alpha$ were upregulated in the lung at the onset of infection, which increased intracellular iron homeostasis, with the net result of increasing levels of total iron and ferritin within the infected lung. Interestingly, the study also indicated that transition from an acute to a stable condition corresponded with a decrease in the total iron and ferritin levels (Reid et al., 2004). However, patients with clinical decline had no associated decrease in iron and ferritin levels. Taken together, these studies show that there is sufficient ferritin in the lung to act as a potential source of iron for bacteria able to utilize it, and that the inflammatory response in the CF lung further elevates the available ferritin-bound iron.

Previous studies have shown that bacteria can acquire iron from a number of iron-binding proteins, including transferrin, lactoferrin, haemopexin, haemoglobin, haemoglobin and haemoglobin/haptoglobin complex, and human serum albumin (Morton \& Williams, 1989; Morton \& Stull, 2004; Jin et al., 1999; Stull, 1987). However, no pathogens have been reported to directly acquire iron from ferritin. Evidence suggests indirect, host-mediated effects. For example, Neisseria meningitidis accelerates the degradation of ferritin via modulation of the host-cell machinery (Larson et al., 2004).

To further understand host-pathogen interaction in the lung, we examined the ability of ferritin, at the physiological levels observed in CF patients, to support growth of $B$. cenocepacia $\mathrm{J} 2315$, and examined the molecular mechanism involved in iron acquisition from this metalloprotein complex.

\section{METHODS}

Bacterial strains and growth. B. cenocepacia strains J2315 (BcJ2315) (ATCC BAA-245), Pc 76 (ATCC 17765), Pc77 (ATCC 25608), Pc78 (ATCC 25609), Pc79 (ATCC 25610), Pc83 (ATCC 35130) and Pc84 (ATCC 35254) were obtained from the American Type Culture Collection, and isolates Pc856 (LMG 12614) and Pc857 (LMG 12615) were obtained from the Laboratorium voor Mikrobiologie (LMG). B. cenocepacia isolates K56-2, Bc7 and AU1051 were kindly supplied by P. Sokol, University of Calgary, J. Goldberg, University of Virginia, and J. LiPuma, University of Michigan, Ann Arbor, respectively. All B. cenocepacia isolates were routinely maintained on Luria-Bertani (LB) agar plates. H. influenzae strain Rd KW20 (ATCC 51907), used as a control in some experiments, was routinely maintained on chocolate agar with bacitracin $(\mathrm{BBL})$ at $37^{\circ} \mathrm{C}$.

Iron sources and protease inhibitors. Human and equine ferritin, and equine haemin, were purchased from Sigma. Biotinylated ferritin was purchased from EY laboratories. Haemin was prepared as a $1 \mathrm{mg} \mathrm{ml}^{-1}$ stock solution, as described elsewhere (Poje \& Redfield, 2003). 
Ethylenediamine- $N, N^{\prime}$ bis ( $o$-hydroxyphenyl)acetic acid (EDDHA) was deferrated and prepared as described by Rogers (1973). Protease inhibitors were purchased from Sigma. Protease inhibitor cocktail (Sigma, product no. P2714), containing a mixture of water-soluble protease inhibitors with broad specificity for the inhibition of serine, cysteine, aspartic and metalloproteases, contained 4-(2-aminoethyl)benzenesulfonyl fluoride (AEBSF), E-64, bestatin, leupeptin, aprotinin and sodium EDTA. The cocktail was resuspended in water to give a final concentration, with respect to leupeptin, of $10 \mu \mathrm{M}$. Individual protease inhibitors were purchased from Sigma and suspended in water to final concentrations of $2.0 \mathrm{M}$ AEBSF, $1.30 \mathrm{mM}$ bestatin, $1.4 \mathrm{mM}$ E64, $1.0 \mathrm{mM}$ leupeptin and $300 \mu \mathrm{M}$ aprotinin. Each stock solution was filter-sterilized prior to use. A standard inoculum of BcJ2315 was prepared in LB/EDDHA with $10 \mu \mathrm{g}$ ferritin $\mathrm{ml}^{-1}$ and divided into several $4 \mathrm{ml}$ aliquots, to which 25,50 or $100 \mu \mathrm{l}$ of the protease cocktail was added, and samples were then analysed for growth (see Methods, Growth curves, below). In addition, a similar set of samples was prepared with the non-proteinaceous iron source haem $\left(10 \mu \mathrm{g} \mathrm{ml}^{-1}\right)$ and compared to ferritin as sole iron source. In experiments examining the individual protease inhibitors, $4 \mathrm{ml}$ aliquots of $\mathrm{BcJ} 2315$ were prepared and the inhibitors added in 1,5 and $10 \mu$ quantities.

Growth curves. Growth studies were performed as follows, using the Bioscreen C Microbiology Reader (Oy Growth Curves AB). $\mathrm{BcJ} 2315$ was grown for $12-14 \mathrm{~h}$ on LB agar and used to inoculate $10 \mathrm{ml} \mathrm{LB}$ broth containing $100 \mu \mathrm{M}$ EDDHA, which was incubated for $4 \mathrm{~h}$ at $37^{\circ} \mathrm{C}$ with shaking (175 r.p.m.). Preliminary studies indicated that BcJ2315 was iron starved in LB broth with the addition of $100 \mu \mathrm{m}$ EDDHA. The $4 \mathrm{~h}$ cultures were pelleted by centrifugation and resuspended to an $\mathrm{OD}_{605}$ of 0.5 in fresh LB broth. One millilitre of the bacterial suspension was diluted with $5 \mathrm{ml} \mathrm{LB}$, and this final bacterial suspension was used to inoculate fresh LB $(0.1 \%$, $\mathrm{v} / \mathrm{v}$, inoculum) to give an approximate initial concentration of 200000 c.f.u. $\mathrm{ml}^{-1}$ in media containing various defined supplements. Growth curves were performed in $300 \mu \mathrm{l}$ volumes with five replicates for each growth condition in each individual experiment. Experiments were repeated a minimum of three times. $\mathrm{OD}_{600}$ measurements were obtained at $30 \mathrm{~min}$ intervals with the Bioscreen $\mathrm{C}$ set to incubate at $37^{\circ} \mathrm{C}$ with constant shaking (machine setting at 'low'). Growth curves with $H$. influenzae were performed identically, except that the inoculum was prepared as previously described (Morton et al., 2004). For each condition examined, growth curves were repeated a minimum of three times. Data from a single experiment (mean of five replicates) are displayed in each figure.

Ferritin degradation assay. A standard inoculum of BcJ2315 was prepared as described above and used to seed a $60 \mathrm{ml}$ culture of LB. Following $48 \mathrm{~h}$ growth, the bacteria were pelleted and the culture media filter-sterilized. Biotinylated horse ferritin was added to a final concentration of $5 \mu \mathrm{g} \mathrm{ml}^{-1}$ and the culture split into three aliquots of $20 \mathrm{ml}$. One aliquot was immediately processed to capture the biotinylated ferritin by mixing with $25 \mu \mathrm{l}$ streptavidin-linked agarose beads, with shaking for $1 \mathrm{~h}$. The beads were pelleted by centrifugation for $5 \mathrm{~min}$ at $13000 \mathrm{~g}$, the supernatant was aspirated, and the beads were washed once with $1 \mathrm{ml}$ PBS. After a second centrifugation to collect the beads, the supernatant was removed and the beads were mixed with $25 \mu \mathrm{l}$ SDS-PAGE loading buffer prior to applying the sample to an SDS-PAGE gel. To one of the remaining two samples, AEBSF was added to a final concentration of $200 \mu \mathrm{M}$, and an equal volume of sterile water was added to the remaining sample. The latter two samples were incubated at $37^{\circ} \mathrm{C}$ for $48 \mathrm{~h}$, after which time biotinylated ferritin, and any degradation products, were captured as described above. All samples were analysed in adjacent wells of a $12 \%$ polyacrylamide gel.

Comparative genomics. We examined the preliminary BcJ2315 predicted genes (http://www.sanger.ac.uk/Projects/B_cenocepacia/) for putative extracellular serine proteases. The analysis was performed using the program HMMER (http://hmmer.wustl.edu) to search the Pfam sequence database (Bateman et al., 2004). In particular, the search focused on proteins within the Peptidase_S8 family (PF00082), which includes a number of well-described extracellular serine proteases related to subtilase from Bacillus subtilis (Smith et al., 1966). Predicted proteins with significant matches to serine protease families were then examined to determine whether an autotransporter domain (PF03797) or a signal peptide (http://www.cbs.dtu.dk/services/SignalP/) was present.

Statistical comparisons. The Kruskal-Wallis test was used to compare growth between separate conditions. In some cases the analysis was performed over the entire growth curve and in others only over the period of active growth, as specified above. Analyses were performed using Analyse-It for Microsoft Excel, version 1.71 (Analyse-It Software). $P<0.05$ was considered statistically significant.

\section{RESULTS AND DISCUSSION}

\section{Selection of iron-starved media}

Initial experiments were performed to establish a suitable medium for the growth of $B$. cenocepacia that could be manipulated to provide iron-replete and -deplete growth conditions. Previously we have grown $B$. cenocepacia in LB broth, and this was used with increasing concentrations of EDDHA as an iron chelator. Since BcJ2315 produces siderophores and utilizes siderophore-bound iron, it was not clear if this chelator would limit iron availability within the media. To avoid variation in iron content among different batches of LB, a single large batch of LB broth was prepared and used throughout these experiments. A standard inoculum of BcJ2315 was prepared and used to inoculate LB broth containing 100, 200, 400, 600, 800 and $1000 \mu \mathrm{g}$ EDDHA ml ${ }^{-1}$, and the growth profile determined over a $40 \mathrm{~h}$ incubation period (Fig. 1). An EDDHA concentration of $600 \mu \mathrm{g} \mathrm{ml}^{-1}$ inhibited the growth rate and final concentration of BcJ2315 in LB broth sufficiently to permit the assessment of the utilization of any potential exogenous iron sources. To ensure that the depression of growth by EDDHA was not due to toxic effects, the experiment was repeated with $150 \mathrm{mM}$ iron $\left(\mathrm{FeCl}_{2}\right)$ added to $\mathrm{LB}$ with $600 \mu \mathrm{g}$ EDDHA ml ${ }^{-1}$ and the growth of BcJ2315 observed. The resulting growth curves (data not shown) indicated that addition of the supplemental iron source returned growth to the same level as that observed in EDDHA-free media. Thus, a concentration of $600 \mu \mathrm{g}$ EDDHA ml ${ }^{-1}$ was chosen for all further growth studies.

\section{Growth of B. cenocepacia in media containing ferritin as sole available iron source}

To evaluate the ability of BcJ2315 to utilize ferritin as sole iron source, we compared growth profiles in $\mathrm{LB}, \mathrm{LB} /$ EDDHA and LB/EDDHA containing ferritin. Both human and horse ferritin were examined. Following analysis of the data, it was apparent that both human and horse ferritin supported identical growth of BcJ2315 (data not shown). Due to the ready availability of horse ferritin, and the high degree of homology with human ferritin (87 and 91\% identity of light and heavy chains, respectively), horse 


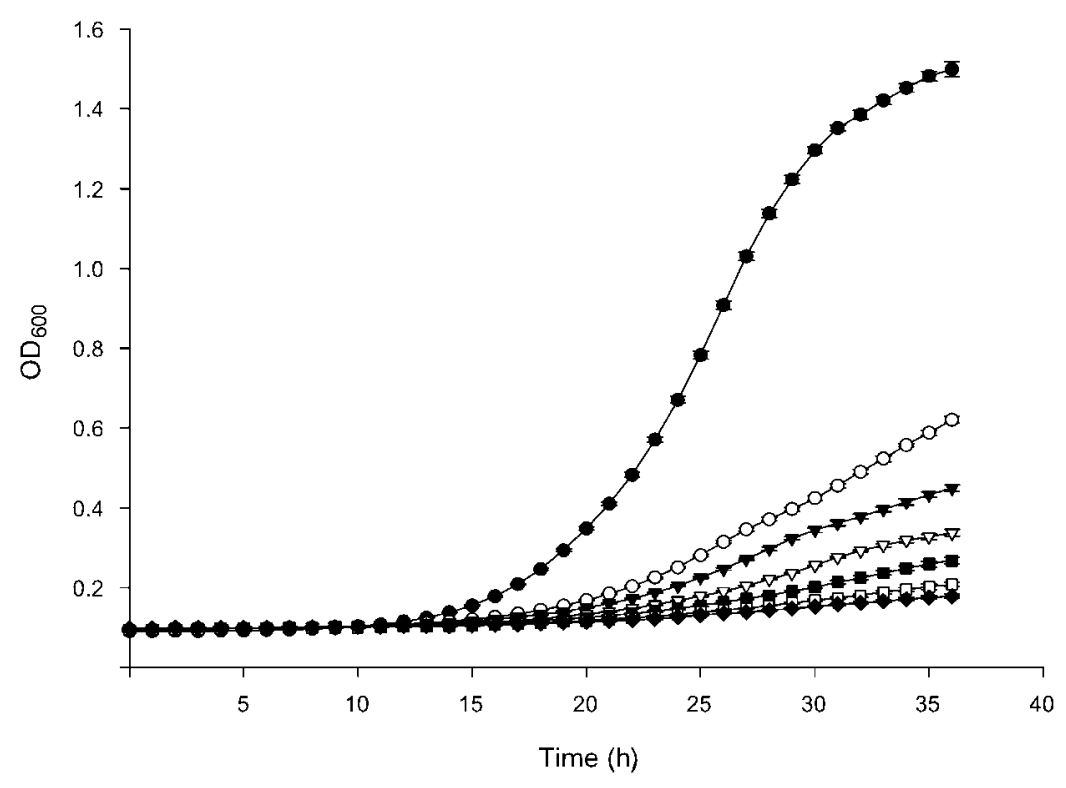

Fig. 1. Effect of EDDHA on the growth of BcJ2315. Growth in LB (O) and LB containing EDDHA at: $100 \mu \mathrm{g} \mathrm{ml}^{-1}(\bigcirc)$, $200 \mu \mathrm{g} \mathrm{ml}^{-1}(\nabla), 400 \mu \mathrm{g} \mathrm{ml}^{-1}(\nabla), 600 \mu \mathrm{g}$ $\mathrm{ml}^{-1}(\boldsymbol{\square}), 800 \mu \mathrm{g} \mathrm{ml}^{-1}(\square)$ or $1000 \mu \mathrm{g} \mathrm{ml}^{-1}$ $(\diamond)$. Error bars represent the standard deviation of the values from five duplicate wells. ferritin was used for the detailed studies of concentration dependence and mechanism of utilization. Fig. 2 shows the growth profiles of BcJ2315 grown in the presence of various concentrations of horse ferritin as sole iron source. From these data it is clear that exogenous ferritin can be utilized by $\mathrm{BcJ} 2315$ as a sole iron source to support growth. At physiological levels of ferritin found in healthy human lungs $\left(0 \cdot 1 \mu \mathrm{g} \mathrm{ml}^{-1}\right)$ there was no apparent growth (data not shown). Examination of the resulting curves indicated that both maximal growth rate and maximal cell yield at the highest concentration of ferritin examined $\left(20 \mu \mathrm{g} \mathrm{ml}^{-1}\right)$ were significantly reduced compared with iron-replete LB media. In addition, we observed an apparent extension of the lag period prior to growth. These experiments were repeated with a further 12 distinct isolates of $B$. cenocepacia. Isolates Pc76, Pc78, Pc79, Pc83, Pc856, Pc857 (LMG 12615), K56-2, Bc7 and AU1051 all showed repression of growth by $600 \mu \mathrm{g}$ EDDHA ml ${ }^{-1}$ which was reversed by the addition of $10 \mu \mathrm{g}$ ferritin $\mathrm{ml}^{-1}$ (data not shown). Using these conditions, there was no growth with isolates Pc77 and Pc84. Further studies with the latter isolates indicated that they were more sensitive to the EDDHA concentration. Experiments repeated with $150 \mu \mathrm{g}$ EDDHA ml ${ }^{-1}$ showed a decrease in growth profiles and also showed that the growth rate was elevated by the addition of ferritin. In all cases, growth curves were similar to those determined for BcJ2315, shown in Fig. 2. Thus, all isolates of B. cenocepacia studied were able to use ferritin as a sole iron source.

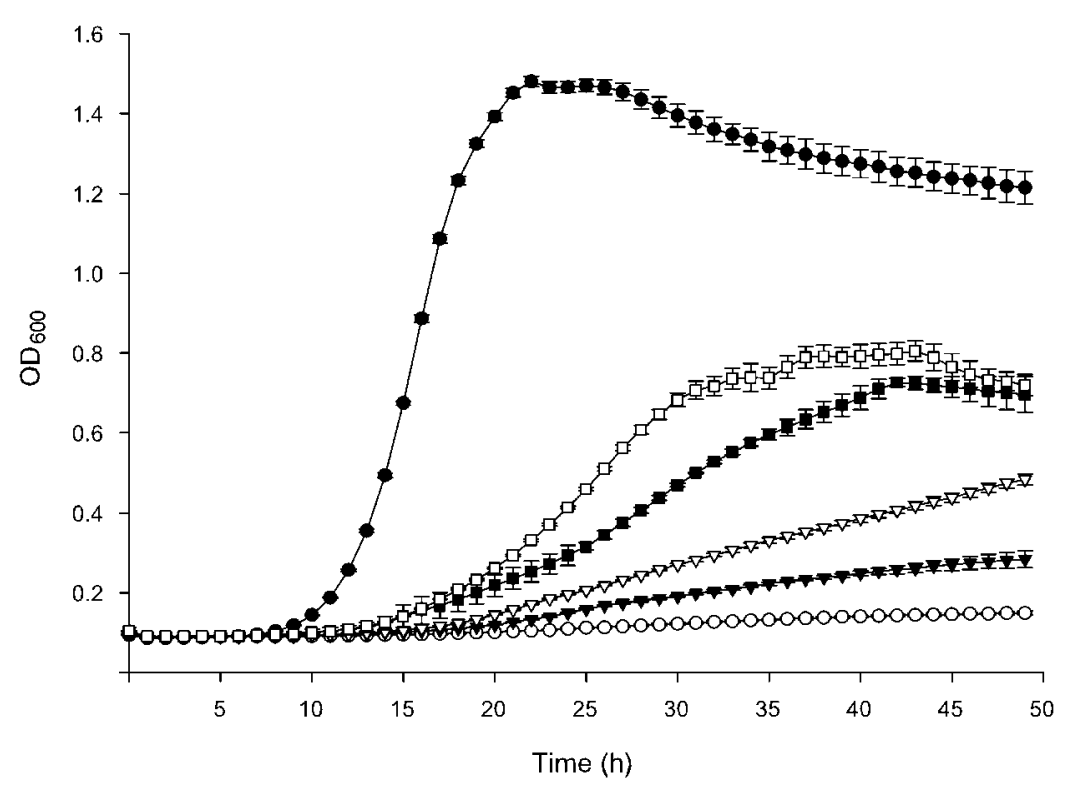

Fig. 2. Growth of $\mathrm{BcJ} 2315$ with ferritin as the sole iron source. Growth in LB $(\bullet)$, LB/EDDHA ( $\bigcirc$ ), and LB/EDDHA containing horse ferritin at: $2.5 \mu \mathrm{g} \mathrm{ml}^{-1}(\boldsymbol{\nabla}), 5.0 \mu \mathrm{g}$ $\mathrm{ml}^{-1}(\nabla), 10 \cdot 0 \mu \mathrm{g} \mathrm{ml}^{-1}(\boldsymbol{\square})$ or $20.0 \mu \mathrm{g} \mathrm{ml}^{-1}$ $(\square)$. Error bars represent the standard deviation of the values from five duplicate wells. 
To ensure that the perceived growth was entirely due to utilization of the iron contained within ferritin, and not due to iron contamination or ferritin autolysis, control experiments using $H$. influenzae were performed. $H$. influenzae strain Rd KW20 was unable to grow in brain heart infusion broth supplemented with protoporphyrin IX and ferritin as the sole iron source. This demonstrated that the ferritin was not contaminated with free iron, nor was it undergoing autolysis (data not shown).

\section{B. cenocepacia obtains iron from ferritin by proteolysis}

To further define the ability of BcJ2315 to utilize ferritin we examined the possible mechanism of acquisition. It is known that $\mathrm{BcJ} 2315$ produces siderophores that may assist in iron assimilation; however, it is unlikely that siderophores penetrate ferritin to release iron. One possible scenario is that the ferritin molecule is physically degraded by a proteolytic process. To examine this mechanism of iron release, we attempted to block ferritin-iron utilization by BcJ2315 using a broad-range protease inhibitor cocktail containing AEBSF, EDTA, bestatin, E64, leupeptin and aprotinin that targets serine, cysteine and metalloproteases. To control for possible toxic effects of the inhibitor cocktail, we also examined the growth of BcJ2315 in LB/EDDHA with haem as sole iron source. Previously, we have shown that haem satisfies the $B$. cenocepacia iron requirement (P. W. Whitby and others, unpublished observations); accordingly, haem was used, since it represents a non-proteinaceous iron source. The protease inhibitor cocktail had no significant effect on BcJ2315 growth in haem-supplemented conditions ( $P=0 \cdot 66, P=0.38$ or $P=0 \cdot 12$ for haem supplemented with 25,50 or $100 \mu \mathrm{l}$ protease inhibitor cocktail, respectively). However, a statistically significant decrease in the growth of BcJ2315 with $10 \mu \mathrm{g}$ ferritin ml ${ }^{-1}$ as the sole iron source was observed $(P=0.039$ for $25 \mu$ and $P<0.0001$ for 50 and $100 \mu \mathrm{l}$ protease inhibitor cocktail) (Fig. 3). Examination of the resulting curves indicated that the lag phase increased with increasing concentration of protease inhibitor cocktail, although the growth rate was unaffected. These results suggest that the cocktail prevented BcJ2135 from using ferritin-bound iron until a threshold level of de novo synthesized protease was achieved. Alternatively, ferritin may have been unstable under our conditions and EDTA present in the protease cocktail may have bound any released iron and changed the growth profiles. To examine the latter possibility, BcJ2315 was grown in LB/EDDHA/ ferritin with an EDTA concentration equivalent to that used in the protease cocktail. No significant impact on the growth profile was observed (data not shown). Together these findings strongly suggest that the utilization of ferritin iron by $\mathrm{Bc}$ 2315 is protease-mediated.

To further investigate the apparently protease-dependent utilization of ferritin by $\mathrm{BcJ} 2315$, the effect of the specific protease inhibitors in the cocktail was examined. As in the experiments described above, non-specific effects of the protease inhibitors were also determined for BcJ2315 grown in LB/EDDHA supplemented with haem. None of the protease inhibitors showed an effect on growth in LB/EDDHA supplemented with haem across the concentrations examined (data not shown). With the exception of AEBSF, none of the inhibitors showed any effect on the growth profiles in media supplemented with ferritin as the sole iron source (data not shown). In contrast, increasing concentrations of AEBSF abrogated the ability of BcJ2315 to grow in medium supplemented with ferritin as iron source in a dose-dependent manner (Fig. 4). AEBSF is an irreversible inhibitor of serine proteases, and is stable in aqueous solution. Thus, the growth curves obtained using this compound are consistent with the inhibition of de novo synthesized protease until the level of protease inhibitor in

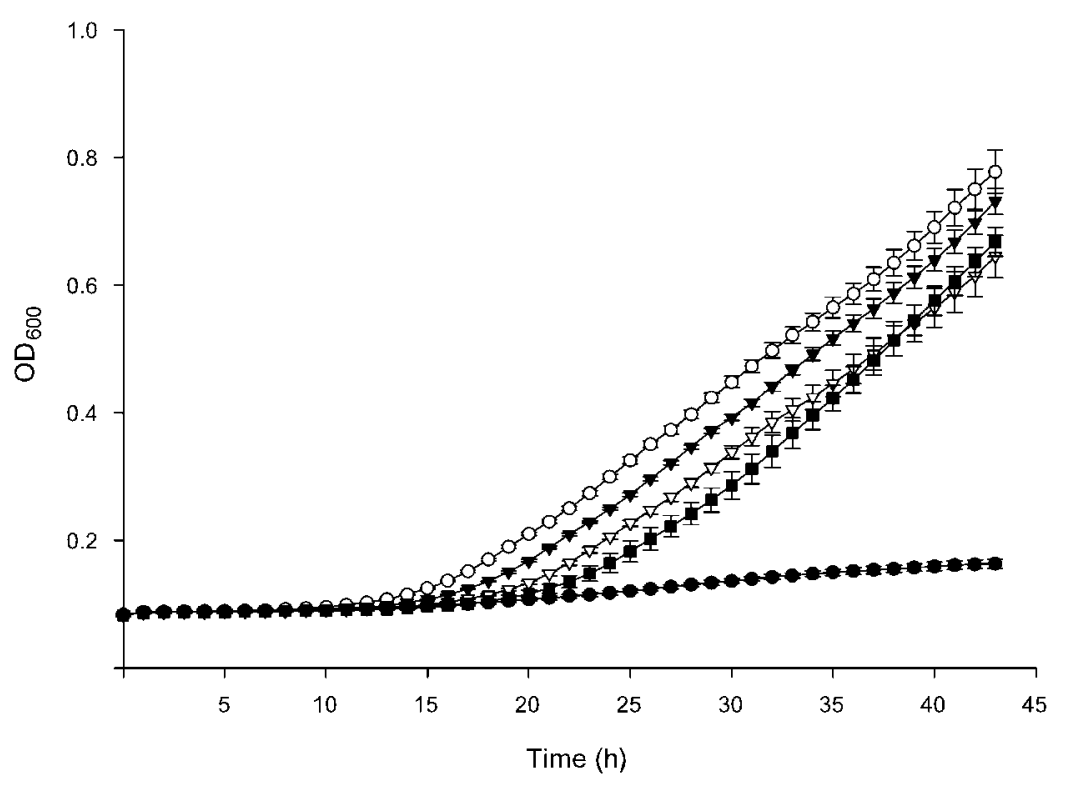

Fig. 3. Effect of a protease inhibitor cocktail on ferritin utilization by BcJ2315. Growth in LB/EDDHA (๑), LB/EDDHA/ferritin ( $\bigcirc)$, and LB/EDDHA/ferritin containing protease inhibitor cocktail solution at: $6 \cdot 3 \mu \mathrm{ml}^{-1}(\boldsymbol{\nabla})$, $12.5 \mu \mathrm{ml}^{-1}(\nabla)$ or $25 \mu \mathrm{ml}^{-1}(\boldsymbol{\square})$. Error bars represent the standard deviation of the values from five duplicate wells. 


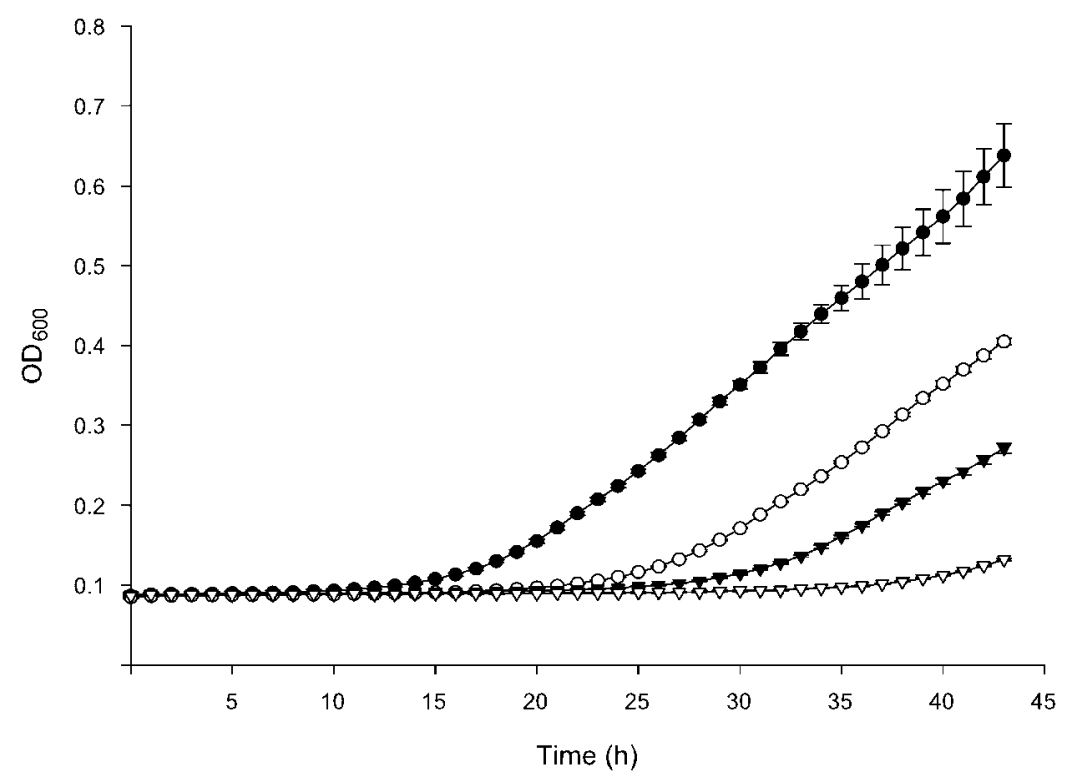

Fig. 4. Effect of AEBSF on ferritin utilization by BcJ2315. Growth in LB/EDDHA/ferritin (O), and LB/EDDHA/ferritin containing AEBSF at: $50 \mu \mathrm{M}(\bigcirc), 250 \mu \mathrm{M}(\boldsymbol{\nabla})$ or $500 \mu \mathrm{M}(\nabla)$. Error bars represent the standard deviation of the values from five duplicate wells. the medium is exhausted, at which point the bacteria utilize the available ferritin to satisfy their growth requirements.

To further examine the possibility of proteolytic cleavage of ferritin by BcJ2315, the effect of incubation of biotinylated ferritin with a growing culture of BcJ2135 over a 4-day period was analysed. Biotinylated ferritin was chosen for these experiments because this tag, on intact ferritin and/ or its degradation products, facilitates purification using streptavidin-linked agarose beads. We first established that biotinylated ferritin supported growth of BcJ2315 in a manner similar to non-biotinylated ferritin (data not shown). Following purification of the biotinylated ferritin and any potential degradation products over a 4-day period, the samples were analysed on a $12 \%$ polyacrylamide gel and stained with Gelcode Blue Stain reagent (Pierce). Examination of the destained gel (Fig. 5) showed two distinct bands for the time zero sample. The lower band corresponded to approximately $20 \mathrm{kDa}$, the size of the ferritin subunits. The higher band presumably corresponded to native intact ferritin composed of 24 subunits, although this band did not correspond to the predicted molecular mass of the complex. This may reflect the partial denaturation of the complex and lack of resolution of the gel in this region. It is known that ferritins are resistant to high temperature, and the 5 min boiling step employed in sample preparation is probably insufficient to dissociate all the ferritin macromolecules. Examination of the band corresponding to the ferritin subunits showed a distinct decrease in band intensity over the duration of the experiment. Similarly, the high-molecular-mass complex showed a corresponding decrease in intensity with incubation time. Examination of the remainder of the gel indicated multiple, discreet degradation products located between the high- and low-molecular-mass bands that peaked in the $72 \mathrm{~h}$ sample. These may represent partially intact complexes that have undergone limited proteolysis. The intensity of these bands decreased in the $96 \mathrm{~h}$ sample; however, a smaller, more diffuse band appeared at the bottom of the gel in this sample, corresponding to small fragments of protein. Control samples, containing sterile LB incubated over the same time period, showed no differences in the banding patterns over a 4-day period (data not shown). This experiment clearly shows the proteolytic degradation of ferritin by a growing culture of $\mathrm{BcJ} 2315$. To determine if the protease is

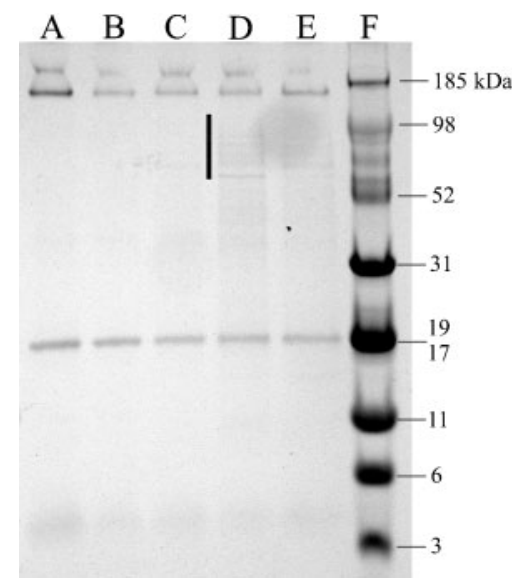

Fig. 5. Proteolytic degradation of ferritin by $B c J 2315$. Samples $(1 \mathrm{ml})$ of a growing culture of BcJ2315 in LB/EDDHA with $20 \mu \mathrm{g}$ biotinylated ferritin $\mathrm{ml}^{-1}$ were taken at $0,24,48,72$ and $96 \mathrm{~h}$ (lanes A-E, respectively) and the ferritin was purified on agarose beads. Lane $\mathrm{F}$ contains molecular mass markers, sizes as indicated. The dark-staining band at the top corresponds to intact ferritin, while the second major band at approx. $20 \mathrm{kDa}$ represents the subunit. Lanes $\mathrm{D}$ and $\mathrm{E}$ show partial degradation products (denoted with a line alongside lane $\mathrm{D}$ ). 
secreted or cell associated, the experiment was repeated with cell-free supernatant from a $48 \mathrm{~h}$ culture of BcJ2315 grown in LB. In the absence of AEBSF, degradation of ferritin was observed. Neither LB alone nor supernatant with added AEBSF showed ferritin degradation (data not shown), thus indicating that the proteolytic activity is secreted into the growth medium.

These results clearly indicate the role of a serine protease in the acquisition of iron from ferritin by BcJ2315. Presumably, since the EDDHA would bind any iron released into the medium, this must occur at or near the cell surface. With these two points as search criteria, we examined the genome of BcJ2315 to identify surface-associated serine proteases. The results of this study indicated that the genome of BcJ2315 contains four predicted proteins that are candidates for an extracellular serine protease. These are encoded by the putative ORFs BCAM0922, BCAM0957, BCAM1744 and BCAS0405. Each of these predicted proteins contained a putative signal peptide sequence, and comparison with the Pfam protein database revealed that all were homologous to the subtilase family of serine proteases (PF00082). Future studies will be performed to further elucidate the role, if any, of these proteases in the utilization of ferritin-bound iron.

In summary, this study shows that isolates of B. cenocepacia can use iron sequestered in ferritin in a protease-dependent manner. The protease(s) involved is a serine protease and is probably extracellular, being either secreted into the growth media or present on cell surfaces. The ability to use ferritin may contribute to the ability of $B$. cenocepacia to colonize and persist in the CF lung.

\section{ACKNOWLEDGEMENTS}

This work was supported by the grants from the Oklahoma Center for the Advancement of Science and Technology (OCAST) and the Cystic Fibrosis Foundation to P.W.W. We gratefully acknowledge the support of the Children's Medical Research Institute.

\section{REFERENCES}

Bateman, A., Coin, L., Durbin, R. \& 10 other authors (2004). The Pfam protein families database. Nucleic Acids Res 32, D138-D141.

Burkholder, W. H. (1950). Sour skin, a bacterial rot of onion bulbs. Phytopathology 14, 127-130.

Crichton, R. R. (1969). Studies on the structure of ferritin and apoferritin from horse spleen. I. Tryptic digestion of ferritin and apoferritin. Biochim Biophys Acta 194, 34-42.

Darling, P., Chan, M., Cox, A. D. \& Sokol, P. A. (1998). Siderophore production by cystic fibrosis isolates of Burkholderia cepacia. Infect Immun 66, 874-877.

Govan, J. R., Brown, P. H., Maddison, J., Doherty, C. J., Nelson, J. W., Dodd, M., Greening, A. P. \& Webb, A. K. (1993). Evidence for transmission of Pseudomonas cepacia by social contact in cystic fibrosis. Lancet 342, 15-19.

Gutteridge, J. M., Quinlan, G. J. \& Evans, T. W. (2001). The iron paradox of heart and lungs and its implications for acute lung injury. Free Radic Res 34, 439-443.
Hutchison, M. L., Poxton, I. R. \& Govan, J. R. (1998). Burkholderia cepacia produces a hemolysin that is capable of inducing apoptosis and degranulation of mammalian phagocytes. Infect Immun 66, 2033-2039.

Isles, A., Maclusky, I., Corey, M., Gold, R., Prober, C., Fleming, P. \& Levison, H. (1984). Pseudomonas cepacia infection in cystic fibrosis: an emerging problem. J Pediatr 104, 206-210.

Jin, H., Ren, Z., Whitby, P. W., Morton, D. J. \& Stull, T. L. (1999). Characterization of $h g p A$, a gene encoding a hemoglobin/hemoglobinhaptoglobin binding protein of Haemophilus influenzae. Microbiology 145, 905-914.

Johnson, W. M., Tyler, S. D. \& Rozee, K. R. (1994). Linkage analysis of geographic and clinical clusters in Pseudomonas cepacia infections by multilocus enzyme electrophoresis and ribotyping. J Clin Microbiol 32, 924-930.

Kooi, C., Cox, A., Darling, P. \& Sokol, P. A. (1994). Neutralizing monoclonal antibodies to an extracellular Pseudomonas cepacia protease. Infect Immun 62, 2811-2817.

Larson, J. A., Howie, H. L. \& So, M. (2004). Neisseria meningitidis accelerates ferritin degradation in host epithelial cells to yield an essential iron source. Mol Microbiol 53, 807-820.

Lewin, C., Doherty, C. \& Govan, J. R. W. (1993). In vitro activities of meropenem, PD 127391, PD 131628, ceftazidime, chloramphenicol, cotrimoxazole, and ciprofloxacin against Pseudomonas cepacia. Antimicrob Agents Chemother 37, 123-125.

Liu, X. \& Theil, E. C. (2005). Ferritins: dynamic management of biological iron and oxygen chemistry. Acc Chem Res 38, 167-175.

Mahenthiralingam, E., Urban, T. A. \& Goldberg, J. B. (2005). The multifarious, multireplicon Burkholderia cepacia complex. Nat Rev Microbiol 3, 144-156.

Morton, D. J. \& Stull, T. L. (2004). Haemophilus. In Iron Transport in Bacteria, pp. 273-292. Edited by J. H. Crosa, A. R. Mey \& S. M. Payne. Washington, DC: American Society for Microbiology.

Morton, D. J. \& Williams, P. (1989). Utilization of transferrin-bound iron by Haemophilus species of human and porcine origins. FEMS Microbiol Lett 53, 123-127.

Morton, D. J., Smith, A., Madore, L. L., VanWagoner, T. M., Seale, T. W., Whitby, P. W. \& Stull, T. L. (2004). Identification of a haem utilization protein (Hup) in Haemophilus influenzae. Microbiology 150, 3923-3933.

Nakazawa, T., Yamada, Y. \& Ishibashi, M. (1987). Characterization of hemolysin in extracellular products of Pseudomonas cepacia. J Clin Microbiol 25, 195-198.

Pitt, T. L., Kaufmann, M. E., Patel, P. S., Benge, L. C., Gaskin, S. \& Livermore, D. M. (1996). Type characterisation and antibiotic susceptibility of Burkholderia (Pseudomonas) cepacia isolates from patients with cystic fibrosis in the United Kingdom and the Republic of Ireland. J Med Microbiol 44, 203-210.

Poje, G. \& Redfield, R. J. (2003). General methods for culturing Haemophilus influenzae. Methods Mol Med 71, 51-56.

Prince, A. (1986). Antibiotic resistance of Pseudomonas species. J Pediatr 108, 830-834.

Reid, D. W., Lam, Q. T., Schneider, H. \& Walters, E. H. (2004). Airway iron and iron-regulatory cytokines in cystic fibrosis. Eur Respir J 24, 286-291.

Rogers, H. J. (1973). Iron-binding catechols and virulence in Escherichia coli. Infect Immun 7, 445-456.

Rosenstein, B. J. \& Hall, D. E. (1980). Pneumonia and septicemia due to Pseudomonas cepacia in a patient with cystic fibrosis. Johns Hopkins Med J 147, 188-189.

Simpson, I. N., Finlay, J., Winstanley, D. J., Dewhurst, N., Nelson, J. W., Butler, S. L. \& Govan, J. R. W. (1994). Multi-resistance isolates 
possessing characteristics of both Burkholderia (Pseudomonas) cepacia and Burkholderia gladioli from patients with cystic fibrosis. J Antimicrob Chemother 34, 353-361.

Smith, E. L., Markland, F. S., Kasper, C. B., DeLange, R. J., Landon, M. \& Evans, W. H. (1966). The complete amino acid sequence of two types of subtilisin, BPN' and Carlsberg. J Biol Chem 241, 5974-5976.

Sokol, P. A. (1986). Production and utilization of pyochelin by clinical isolates of Pseudomonas cepacia. J Clin Microbiol 23, 560-562.

Stites, S. W., Walters, B., O'Brien-Ladner, A. R., Bailey, K. \& Wesselius, L. J. (1998). Increased iron and ferritin content of sputum from patients with cystic fibrosis or chronic bronchitis. Chest 114, 814-819.

Stites, S. W., Plautz, M. W., Bailey, K., O'Brien-Ladner, A. R. \& Wesselius, L. J. (1999). Increased concentrations of iron and isoferritins in the lower respiratory tract of patients with stable cystic fibrosis. Am J Respir Crit Care Med 160, 796-801.
Stull, T. L. (1987). Protein sources of heme for Haemophilus influenzae. Infect Immun 55, 148-153.

Thomassen, M. J., Demko, C. A., Klinger, J. D. \& Stern, R. C. (1985). Pseudomonas cepacia colonization among patients with cystic fibrosis. A new opportunist. Am Rev Respir Dis 131, 791-796.

Turi, J. L., Yang, F., Garrick, M. D., Piantadosi, C. A. \& Ghio, A. J. (2004). The iron cycle and oxidative stress in the lung. Free Radic Biol Med 36, 850-857.

Vonberg, R. P. \& Gastmeier, P. (2005). Isolation of infectious cystic fibrosis patients: results of a systematic review. Infect Control Hosp Epidemiol 26, 401-409.

Walters, S. \& Smith, E. G. (1993). Pseudomonas cepacia in cystic fibrosis: transmissibility and implications. Lancet 342, 3-4.

Whitby, P. W., VanWagoner, T. M., Taylor, A. A., Seale, T. W., Morton, D. J., LiPuma, J. J. \& Stull, T. L. (2006). Identification of an RTX determinant of Burkholderia cenocepacia J2315 by subtractive hybridization. J Med Microbiol 55, 11-21. 